\title{
WHATSAPP, COMUNICACIÓN MÓVIL Y PARTICIPACIÓN POLÍTICA: UN ESTUDIO CUANTITATIVO EN ECUADOR
}

\author{
MARCOS ZUMÁRRAGA-ESPINOSA* \\ Universidad Politécnica Salesiana \\ mzumarraga@ups.edu.ec
}

Recibido: 7/9/2020 Aceptado: 28/1/2021

doi: https://doi.org/10.26439/contratexto2021.n035.4839

RESUMEN. Este trabajo explora la relación entre distintos usos políticos de WhatsApp y los comportamientos de participación política en el contexto ecuatoriano. Con el propósito de lograr un entendimiento más detallado, la participación política se clasificó en tres modos de acción específicos: offline convencional, offline protesta y online. Por su parte, el uso político de la aplicación se desagregó en tres tipos: (a) consumo de información política, (b) expresión y discusión política, y (c) contacto con grupos políticos online. A partir de los datos de encuestas aplicadas en el Distrito Metropolitano de Quito (Ecuador) en el 2018, los análisis de regresión múltiple desarrollados indican que el consumo de información política vía WhatsApp solo influye significativamente sobre las acciones de protesta política. Por el contrario, la expresión y la discusión política mediante esta aplicación móvil producen efectos movilizadores sobre todas las modalidades de participación analizadas. Efecto consistente que también se observa cuando WhatsApp se emplea para la interacción con grupos políticos online. En el apartado final se discuten los hallazgos del estudio tomando en cuenta la investigación previa en torno a los efectos democráticos de esta aplicación.

PALABRAS CLAVE: WhatsApp / participación política offline / participación política online / comunicación política móvil / Ecuador

\footnotetext{
* Magíster en Gestión Pública por el Instituto de Altos Estudios Nacionales, Ecuador (véase: https://orcid. org/0000-0001-9930-9005).
} 


\section{WHATSAPP, MOBILE COMMUNICATION AND POLITICAL PARTICIPATION: A QUANTITATIVE STUDY FROM ECUADOR}

ABSTRACT. This work explores the relationship between the different political uses of WhatsApp and the political participation behaviors in the Ecuadorian context. With the purpose of achieving a more detailed understanding, political participation was classified into three specific modes of action: offline conventional, offline protest and online. On the other hand, the political use of WhatsApp was classified into three types: a) consumption of political information, b) political expression and discussion, and c) contact with online political groups. Using survey data collected in the Metropolitan District of Quito (Ecuador) in 2018, multiple regression analyses indicate that the consumption of political information via WhatsApp only significantly influences political protest actions. On the contrary, the political expression and discussion through this mobile application produce mobilizing effects on all the analyzed participation forms: a consistent effect also observed when WhatsApp is used for interaction with online political groups. The final section discusses the findings of the study taking into account the previous research on the democratic effects of WhatsApp.

KEYWORDS: WhatsApp / offline political participation / online political participation / mobile political communication / Ecuador

\section{WHATSAPP, COMUNICAÇ̃̃O MÓVEL E PARTICIPAÇÃO POLÍTICA: UM ESTUDO QUANTITATIVO EMNO EQUADOR}

RESUMO. Este trabalho analisa a relação entre diferentes usos políticos do WhatsApp e os comportamentos de participação política no contexto equatoriano. Para um entendimento mais detalhado, a participação política foi classificada em três modos específicos de ação: offline convencional, protesto offline e online. Por sua vez, o uso político do WhatsApp foi dividido em 3 tipos: a) consumo de informação política, b) expressão e discussão política, e c) contato com grupos políticos online. Usando dados de pesquisas aplicadas no Distrito Metropolitano de Quito (Equador) em 2018, as análises de regressão múltipla desenvolvidas indicam que o consumo de informações políticas via WhatsApp influencia significativamente nas ações de protesto político. Contudo, a expressão e a discussão política através desta aplicação móvel produzem efeitos mobilizadores em todas as modalidades de participação analisadas. Efeito consistente que também é observado quando o WhatsApp é usado para interagir com grupos políticos online. A seção final discute os resultados do estudo, levando em consideração pesquisas anteriores sobre os efeitos democráticos do WhatsApp.

PALAVRAS-CHAVE: WhatsApp / participação política offline / participação política online / comunicação política móvel / Equador 


\section{INTRODUCCIÓN}

La evolución del escenario democrático contemporáneo ha estado acompañada por la creciente popularidad y relevancia de las tecnologías de comunicación digital como herramientas de acción y deliberación política (Boulianne, 2015; Fenton y Barassi, 2011). La web 2.0 ha originado plataformas digitales como Facebook, Twitter o YouTube, que permiten a los usuarios ser simultáneamente consumidores y productores de contenidos políticos (Effing et al., 2011), además de posibilitar la expresión de puntos de vista y opiniones políticas a través de publicaciones o conversaciones por chat en espacios altamente interactivos e interconectados (Gil de Zúñiga et al., 2014). En el caso de los activistas, candidatos u organizaciones políticas, las redes sociales reducen el costo y facilitan la difusión a gran escala de información movilizadora, como invitaciones o convocatorias para participar en actividades políticas online u offline (Theocharis et al., 2015).

En el ámbito de la comunicación móvil, las posibilidades clásicas, centradas en la realización de llamadas y el intercambio de mensajes de texto, han experimentado un importante salto hacia adelante gracias al surgimiento del teléfono inteligente o smartphone. Así, aplicaciones móviles de redes sociales como Facebook o Twitter permiten que los usuarios de smartphones accedan a los servicios de estas plataformas, también disponibles vía computadoras portátiles o de escritorio. Por otro lado, aplicaciones de mensajería instantánea (AMI) como WhatsApp, Messenger o Snapchat, nativas de dispositivos móviles, se presentan como una evolución de las funciones iniciales de la telefonía celular, incorporando prestaciones que, en muchos casos, resultan muy similares a aquellas aportadas por las plataformas de redes sociales, especialmente en lo que respecta a publicación de contenidos y puntos de vista, transferencia de información y creación de grupos online (Gazit y Aharony, 2018; Quan-Haase y Young, 2010). Desde una perspectiva política, los usuarios de dispositivos móviles se desenvuelven en un escenario de "intermedialidad", siendo capaces de crear su propia experiencia de comportamiento político digital mediante la integración de varias plataformas, empleando tanto AMI como redes sociales (Valeriani y Vaccari, 2018).

Al abordar las implicaciones democráticas de la comunicación móvil, parte importante de la investigación que ha explorado la relación entre el uso de dispositivos móviles y la participación política lo ha hecho como resultado de análisis focalizados en el rol político de las redes sociales, contemplando la versión móvil de estas plataformas al momento de operacionalizar sus distintos usos (Chan et al., 2017; Hopke et al., 2016; Pang, 2018; Yamamoto et al., 2015). Sin embargo, la literatura reciente sobre AMl y comportamiento político ha resaltado la necesidad de estudiar de manera más específica estas tecnologías de comunicación móvil, puesto que ofrecen posibilidades diferenciadas con respecto a las redes sociales (Valenzuela et al., 2019). Si bien tanto las AMI como las aplicaciones móviles de redes sociales posibilitan que las dinámicas de comunicación política se desarrollen durante el movimiento físico (distinguiéndolas de otros canales 
como, por ejemplo, la comunicación mediada por computadora, telefonía fija, etcétera) (Campbell y Kwak, 2011), las primeras poseen una clara orientación conversacional, además de configurar espacios de comunicación más privados, cerrados y controlados (Gil de Zúñiga et al., 2019; Valeriani y Vaccari, 2018).

WhatsApp es en la actualidad la AMI más utilizada alrededor del mundo (We Are Social y Hootsuite, 2020), que se caracteriza por brindar funciones de conversación, mensajería instantánea y publicación de contenidos multimedia mediante el formato de "estados" o listas de difusión. Los usuarios también cuentan con la alternativa de crear o ser añadidos a grupos para el tratamiento de temáticas específicas (WhatsApp, 2020). Al igual que el resto de las $\mathrm{AMI}$, WhatsApp proporciona condiciones de comunicación más controladas, que favorecen una interacción más selectiva con usuarios individuales y grupos claramente definidos, con prestaciones como conocer quiénes han leído los mensajes enviados y en qué momento, tiempos limitados para la visibilidad de los estados publicados, entre otras (Campbell y Kwak, 2011; Karapanos et al., 2016; Valeriani y Vaccari, 2018). De esta manera, los usuarios pueden dimensionar de modo más eficaz las audiencias que estarán expuestas a sus mensajes. Asimismo, existe una clara preferencia hacia esta plataforma en términos conversacionales, pues se considera como un medio más directo y fácil para mantenerse en contacto, intercambiar mensajes y discutir sobre distintas cuestiones con otros (Church y De Oliveira, 2013; Rubio-Romero y Perlado, 2015). Por último, resalta el papel que se ha asignado a WhatsApp como un mecanismo para mantener contacto frecuente con amigos, familiares y relaciones cercanas, emulando la función de aseguramiento, ligada a confirmar que vínculos cercanos y seres queridos se encuentran bien, la cual es propia del uso del teléfono tradicional (Campbell y Kwak, 2010). Al respecto, el estudio realizado por Church y De Oliveira (2013) sostiene que la utilización de WhatsApp promueve el sentido de comunidad entre sus usuarios. Esto puede explicarse debido a la interacción más cercana y personal que se asigna a esta plataforma, lo cual fomenta el sentimiento de conexión y pertenencia a un círculo social específico. Este efecto tiende a reforzarse gracias a la función de crear y unirse a grupos, que hace posible que los usuarios se mantengan en contacto con múltiples comunidades a la vez (O'Hara et al., 2014).

No obstante, a pesar de la relevancia que WhatsApp ha alcanzado en el plano de la comunicación móvil, particularmente entre los más jóvenes, los aportes investigativos respecto a su impacto político siguen siendo relativamente más escasos en comparación con la literatura sobre redes sociales'. Situando el análisis en la relación

1 Escenario que contrasta con el auge que, durante los últimos años y a nivel mundial, viene caracterizando al uso de AMI como WhatsApp para el consumo de noticias, en tanto que redes sociales como Facebook o Twitter han presentado un declive o estancamiento en dicho rubro. A partir del 2018, lugares como América Latina y Asia destacan por un fuerte posicionamiento de WhatsApp como fuente de consulta para noticias e información de naturaleza política (Newman et al., 2018). 
entre comunicación política móvil y participación política, resulta importante señalar que la investigación previa sobre medios de comunicación ha revelado de manera muy consistente el siguiente patrón: la forma en que el medio se usa importa, y los usos de carácter político favorecen el involucramiento individual en la esfera política de la vida social (Campbell y Kwak, 2010). Esto se ha demostrado empíricamente para medios como la televisión (Shah et al., 2001), la prensa escrita (Scheufele y Nisbet, 2002), la radio (Hollander, 1995), la internet (Shah et al., 2005; Shah et al., 2007) y las redes sociales (Boulianne, 2015; Skoric et al., 2016). En el caso de WhatsApp, el trabajo de Gil de Zúñiga et al. (2019) encontró, a partir de evidencia empírica longitudinal e internacional, que la discusión política a través de esta plataforma influye positivamente en la participación política offline de tipo convencional y protesta. Además, el estudio de Valenzuela et al. (2019), en el contexto chileno, marca entre sus hallazgos una relación positiva entre la participación política offline y el uso de WhatsApp para enviar mensajes políticos y compartir noticias sobre acontecimientos de actualidad. Los efectos de WhatsApp en la participación política online, entendida en sentido amplio (actividades políticas realizadas a través de internet de modo general), aún no se han indagado lo suficiente. En cuanto a sus usos políticos, queda por explorar la correspondencia entre categorías de uso político, como el consumo de información política, y la vinculación con grupos online de naturaleza política.

Con base en lo expuesto, el presente trabajo se propone, como objetivo general, estudiar la relación entre el uso político de WhatsApp y las conductas de participación política a nivel individual. Explorando, de modo más específico, el efecto de tres categorías de uso político de esta AMI: (a) informacional, (b) expresivo/discusión interpersonal y (c) contacto con grupos políticos online. Asimismo, la participación política se aborda en sus tres modalidades: offline convencional, offline protesta y online, con la finalidad de identificar posibles diferencias en el modo en que los patrones de uso político examinados se relacionan con cada tipo de activismo político. Asimismo, el caso de estudio abordado corresponde al contexto ecuatoriano, con información del Distrito Metropolitano de Quito (DMQ).

En Ecuador, WhatsApp ha experimentado un importante posicionamiento como canal de comunicación digital para la ciudadanía. Según datos del Barómetro de las Américas (2018/2019) para el caso ecuatoriano (Moncagatta et al., 2020), es la segunda plataforma digital con mayor cantidad de usuarios en la población en edad de votar, es decir, 16 años o más (60,2 \%), siendo superada únicamente por Facebook (66,9\%). Este escenario contrasta con el panorama regional de América Latina y el Caribe, en donde WhatsApp se sitúa como la plataforma más usada (64,4\%). Cabe destacar que, en términos de frecuencia de uso, supera a Facebook y Twitter, siendo utilizada a diario por un $82,3 \%$ de sus usuarios. Por otra parte, el 33,4\% de los usuarios de WhatsApp emplea esta plataforma para ver información política de manera regular (entre diariamente y 
algunas veces a la semana), ubicándose ligeramente por arriba del 32,1 \% registrado a nivel regional.

\section{REVISIÓN DE LITERATURA}

Como se ha señalado previamente, la investigación en torno a los antecedentes y los efectos del uso político de las AMI sigue siendo escasa. No obstante, existe un amplio repertorio de estudios sobre el impacto de la comunicación digital en el comportamiento político individual, los cuales han situado el foco de atención en redes sociales como Facebook o Twitter (Boulianne, 2020). En este sentido, y tomando en cuenta las particularidades de WhatsApp, se aprovechará el conocimiento acumulado por la literatura sobre redes sociales para abordar teóricamente las relaciones de interés que el presente trabajo se ha propuesto explorar.

Los hallazgos metanalíticos efectuados hasta el momento han mostrado que la capacidad de movilización política de las plataformas digitales depende en buena medida del tipo de actividades que realicen sus usuarios. En consecuencia, aquellos usuarios que emplean sus redes sociales de manera política cuentan con una mayor predisposición para adoptar conductas de participación política (Boulianne, 2015; Boulianne y Theocharis, 2020; Skoric et al., 2016). Los usos políticos que han sido abordados en gran parte de la literatura incluyen el consumo de información política y noticias duras, la expresión de opiniones políticas y la discusión con otros sobre cuestiones políticas o de interés público (Baek, 2015; Campbell y Kwak, 2011; Skoric y Zhu, 2016; Valenzuela et al., 2012; ZumárragaEspinosa et al., 2017). Varios trabajos han profundizado en las interacciones entre estas actividades detectando que tanto la expresión como la discusión política pueden operar como mediadores (e incluso moderadores) que refuerzan la relación entre el uso informacional de las redes sociales y la participación (Cho et al., 2009; Gil de Zúñiga et al., 2014; Jung et al., 2011; Yamamoto et al., 2015). Aunque sus efectos se han estudiado en menor medida, las redes sociales también permiten funciones de contacto y seguimiento de agrupaciones políticas y movimientos sociales (Conroy et al., 2012).

La relación entre el uso informacional de los medios de comunicación y el comportamiento político ha sido un tópico ampliamente estudiado en el campo de la comunicación política. Desde la perspectiva teórica de usos y gratificaciones, los medios son empleados de manera consciente por los individuos para cuatro motivos específicos: diversión, utilidad social, identidad personal y vigilancia (Katz y Gurevitch, 1974). El motivo vigilancia se refiere al consumo de información con el propósito de conocer sobre los acontecimientos actuales, los asuntos políticos y las problemáticas de interés público (Ruggiero, 2000). En este sentido, las investigaciones empíricas efectuadas muestran que la búsqueda y revisión de noticias duras se conecta positivamente con aspectos como el compromiso cívico y la participación política. Estos efectos movilizadores han 
podido verificarse en diferentes medios tradicionales, como la televisión o la prensa escrita (Shah et al., 2007). Evidencia reciente indica que el uso informacional de los nuevos medios sociales también estimula el comportamiento político de los usuarios, tanto en el plano offline (Gil de Zúñiga et al., 2014; Valenzuela, 2013; Yamamoto et al., 2015) como online (Park y Karan, 2014). Con respecto a las explicaciones proporcionadas, los hallazgos realizados a partir de diferentes modelos teóricos de mediación comunicacional coinciden en señalar que la influencia de los usos informacionales sobre la participación se produce en parte gracias a la mediación de procesos de razonamiento y aprendizaje político (Eveland, 2001).

Si bien WhatsApp posibilita el intercambio de información entre sus usuarios, la interacción interpersonal tiende a ocurrir principalmente a través de la conversación y la expresión de ideas o puntos de vista sobre múltiples asuntos. Los efectos democráticos de la discusión y la expresión política han sido estudiados desde varios enfoques teóricos. La teoría de las ganancias diferenciales propone que la discusión sobre cuestiones de políticas o de interés público refuerzan los efectos cognitivos del consumo de información y noticias duras (Hardy y Scheufele, 2005). De esta manera, las personas que usan los medios por motivos de información y vigilancia se encuentran más propensas a adoptar conductas políticas si, además, mantienen discusiones políticas con otros de manera frecuente. Según el modelo 0-S-R-0-R (orientación-estímulo-razonamiento-orientación-respuesta) planteado por Cho et al. (2009), la discusión política, sea que ocurra en el mundo real o a través de internet, opera como una forma de razonamiento que pone en marcha procesos de elaboración mental de varios tipos: reflexión sobre los contenidos receptados, anticipación a conversaciones futuras, composición de ideas para ser expresadas, o integración y entendimiento de los temas tratados. Como resultado de estos esfuerzos cognitivos, "los individuos que participan en discusiones interpersonales pueden usar conceptos complejos, hacer conexiones lógicas profundas entre ellos y crear argumentos coherentes y razonados" (Jung et al., 2011, p. 411). Del mismo modo, la discusión política permite entrar en contacto con las opiniones y conocimientos de otras personas, lo cual ayuda a consolidar y replantear el pensamiento político propio (Pingree, 2007; Eveland, 2004). Por lo tanto, la naturaleza deliberativa de la discusión política fortalece las actitudes y el conocimiento político individual, elevando la predisposición para intervenir en acciones políticas (Jung et al., 2011; Shah et al., 2007).

Además de facilitar la discusión con otros, los medios sociales cuentan con condiciones muy favorables para la publicación de opiniones políticas, dotando a los usuarios de una audiencia permanentemente expuesta a sus comentarios y estados (Gil de Zúñiga et al., 2014; Fenton y Barassi, 2011). Esta propiedad hace posible difundir ideas a un costo mínimo y una escala funcional para el tamaño de la lista de contactos del usuario, tomando en cuenta, asimismo, los grupos a los que pertenece (Lupia y Sin, 2003; Theocharis et al., 2015). Posibilidad que, a su vez, puede aprovecharse de manera más controlada en las 
AMI como WhatsApp, gracias a particularidades como la visibilidad por tiempo limitado para las publicaciones realizadas como estados, el hecho de que los comentarios a los estados publicados solo puedan direccionarse de manera privada, la reciente imposibilidad de tomar captura a las publicaciones de otros usuarios, etcétera. En este sentido, la expresión política facilitada por redes sociales y AMI también activa procesos cognitivos y cambios actitudinales que conducen a la participación. Según el modelo de efectos bidireccionales del mensaje formulado por Pingree (2007), la expresión ejerce tres tipos de efectos sobre el emisor: efectos de las expectativas previas a la expresión, efectos de composición del mensaje y efectos de la emisión del mensaje. Estos componentes de la expresión involucran procesos mentales que estimulan la construcción y reconstrucción de pensamientos a partir de nuevas ideas, la creación de autopercepciones compatibles con las ideas expresadas, el compromiso con los puntos de vista generados y la sensación de ser escuchados. En conjunto, los efectos descritos pueden hacer que los individuos que se expresan pasen de ser observadores a participantes activos de la vida política (Gil de Zúñiga et al., 2014). Por último, se espera que la naturaleza textual de las publicaciones en plataformas digitales refuerce el efecto movilizador de la expresión sobre la participación. Esto se debe a que los mensajes escritos demandan mayor esfuerzo de composición (Cho et al., 2009) y propician conversaciones más orientadas hacia objetivos concretos que las comunicaciones verbales (Valenzuela, 2013).

La creación de grupos es una posibilidad presente en la mayoría de redes sociales y $\mathrm{AMI}$; no obstante, la relación entre el uso político de esta herramienta y la participación política es un tema que ha recibido menor atención de parte de la literatura especializada. El estudio efectuado por Conroy et al. (2012) para el caso de Facebook sugiere que los grupos políticos online generan beneficios similares a los grupos offline a la hora de motivar la acción política, proporcionando información y condiciones favorables para que sus miembros se expresen e intercambien puntos de vista sobre diferentes cuestiones sociales o políticas. Por su parte, el rol de los grupos políticos creados en el marco de la aplicación móvil WhatsApp se mantiene aún inexplorado. Aunque los usuarios emplean WhatsApp especialmente para interactuar con su círculo social más cercano, la pertenencia a grupos conformados por otros usuarios puede potenciar los efectos movilizadores de la comunicación política. En primera instancia, el intercambio político entre una persona y sus vínculos fuertes fomenta el compromiso y la participación política (Gil de Zúñiga y Valenzuela, 2011; Wojcieszak, 2009). Sin embargo, existen posturas que afirman que la calidad de la discusión política aumenta a medida que los puntos de vista expresados sean más diversos (Druckman y Nelson, 2003). Esto ocurre gracias a los esfuerzos cognitivos que entran en juego al deliberar con personas que piensan de manera distinta; tal escenario no solo exige mayor dominio sobre los temas tratados y anticipación a posibles reacciones con el fin de formular argumentos más consistentes, sino que también permite entrar en contacto con conocimientos y perspectivas diferentes 
(Eveland, 2004; Mutz, 2002; Pingree, 2007). Dada la alta selectividad social que posibilitan los medios sociales, la interacción directa de los usuarios se limita en mayor medida a aquellos usuarios con opiniones y formas de pensar similares. Esta tendencia hacia la homogeneidad podría resultar perjudicial para el aprendizaje y el desarrollo de la postura política individual (Campbell y Kwak, 2011). Ante esto los grupos políticos online ejercen un efecto compensador que incrementa las oportunidades de que una persona se exponga a puntos de vista más diversos, dado su limitado control sobre las expresiones generadas por el resto de usuarios (Bakshy et al., 2015). En consecuencia, se esperaría que mientras mayor sea la frecuencia con la que un individuo se une e interviene en grupos políticos online, mayor será su predisposición a participar en actividades políticas.

Considerando los distintos tipos de conductas políticas que pueden desplegarse a través de medios sociales, la reciente literatura sobre WhatsApp ha centrado su atención principalmente en los efectos movilizadores producidos por el uso de esta AMI para fines como la divulgación de información política y la discusión política interpersonal (Gil de Zúñiga et al., 2019; Valenzuela et al., 2019). En contraste, posibilidades de WhatsApp como el consumo de información política o la vinculación a grupos online con orientación política, y su relación con la participación política, aún no se han explorado lo suficiente.

\section{MODALIDADES DE PARTICIPACIÓN POLÍTICA}

El concepto de participación política engloba aquellas actividades desarrolladas por la ciudadanía con el propósito de influir en el proceso político. En el presente trabajo, el abordaje de la participación política toma en consideración la extensa literatura a favor de una diferencia entre su modalidad online y offline (Gibson y Cantijoch, 2013; Kim et al., 2017; Oser et al., 2013; Nam, 2012). Asimismo, el activismo offline puede subdividirse en acciones de tipo convencional y protesta. La participación convencional hace referencia a las acciones políticas, consideradas como legítimas y normales, que se canalizan a través de los agentes representativos, dinámicas electorales y mecanismos institucionales establecidos por el sistema político (Ekman y Amnå, 2012; Oñate, 2005). En contraste, las formas de protesta política se caracterizan por distanciarse en mayor o menor medida de la participación institucionalizada y los procedimientos democráticos tradicionales, pudiendo incluso rebasar los límites de la legalidad en ciertos casos (DiGrazia, 2014; Somuano, 2005).

Por último, la categoría de participación política online se refiere a las acciones políticas efectuadas a través de internet de manera general, incluyendo formas participativas que cuentan con una contraparte offline (donación de dinero para fines políticos a través de internet, contacto online con actores políticos o funcionarios públicos, promover candidatos u organizaciones políticas mediante el uso de herramientas digitales, etcétera) y conductas políticas nativas del entorno online, como escribir blogs políticos o el 
activismo político ejercido vía redes sociales (Gibson y Cantijoch, 2013). Además, tomando en cuenta la tesis de la "actualización" planteada por Krueger (2002), que aboga por una conceptualización más amplia de la participación política online, también se contemplan actividades políticas de tipo informacional y expresivo. Según la tesis de la "actualización", a pesar de que consumir noticias duras o discutir con otros sobre política pueden considerarse como formas pasivas, o preparticipativas, de involucramiento político en el ámbito offline, la esfera digital permite que dichos actos adquieran un carácter mucho más activo y participativo.

Con base en la revisión de literatura efectuada, y considerando las particularidades contextuales del caso ecuatoriano, el presente trabajo se plantea las siguientes preguntas de investigación:

1. ¿Cómo se relaciona el uso político informacional de WhatsApp con la participación política en sus diferentes modalidades?

2. ¿En qué medida el uso de WhatsApp para la expresión y la discusión política se relaciona con las modalidades de participación política?

3. ¿Cuál es la relación entre el uso de WhatsApp para el contacto con grupos políticos y las formas de participación política?

\section{METODOLOGÍA}

El estudio empírico se efectuó a partir de una muestra de 1520 participantes radicados en el Distrito Metropolitano de Quito (DMQ), Ecuador. Se efectuó un muestreo no probabilístico estableciendo cuotas por género y administración zonal con el propósito de lograr mayor representatividad de la población quiteña. El 49,7 \% de la muestra estuvo compuesto por mujeres. Contemplando las administraciones zonales del DMQ, el proceso de recolección de datos se distribuyó geográficamente según las siguientes cuotas: $14,1 \%$ de los participantes pertenecen a la administración zonal La Delicia, 5,7 \% a Calderón, 22,9 \% a Eugenio Espejo, 12,8 \% a Manuela Sáenz, 20,3 \% a Eloy Alfaro, 10,9 \% a Quitumbe, 7,2 \% a Tumbaco y 6,1 \% a Los Chillos. Dichas cuotas se establecieron de acuerdo con el peso relativo de cada administración zonal en términos de población en edad de votar (16 años o más).

La edad promedio se situó en 31,9 años (DT = 13,0). El grado máximo de estudios que han cursado los participantes presenta la siguiente distribución: 0,3\% sin instrucción formal, $4 \%$ primaria, $10,5 \%$ secundaria, $29,8 \%$ bachillerato, $21,2 \%$ universidad y $3,5 \%$ posgrado. Los ingresos familiares se registraron mediante intervalos que van desde "385 USD o menos" hasta "3474 USD o más"; la moda se ubicó en el segmento de "386 hasta 771 USD" (27,4 \%). Resulta pertinente subrayar que esta muestra se encuentra 
sobrerrepresentada por la población más joven², que es el grupo de edad que precisamente se encuentra más propenso a utilizar WhatsApp en Ecuador. Este aspecto debe tomarse en consideración al evaluar las limitaciones del estudio en cuanto a generalización de resultados, pues, dado el perfil muestral analizado, es más probable que los hallazgos producidos sean representativos del segmento más joven de la ciudadanía.

Los datos utilizados proceden de la Encuesta General de Opinión y Participación Política desarrollada y aplicada periódicamente por el Grupo de Investigaciones Psicosociales de la Universidad Politécnica Salesiana (GIPS-UPS). El registro de información tuvo lugar durante el segundo cuatrimestre del 2018 con la ayuda de estudiantes universitarios. Las encuestas se aplicaron de manera autoadministrada. Los participantes colaboraron en el estudio de manera voluntaria y anónima, siendo debidamente informados sobre los protocolos de confidencialidad para el manejo de los datos recolectados.

\section{Variables dependientes}

En el marco de la participación política offline se realizaron mediciones diferenciadas de la modalidad convencional y protesta. La participación política convencional se evaluó por medio de 8 ítems relacionados con actividades participativas que pueden llevarse a cabo por las vías institucionales y representativas que normalmente están disponibles en regímenes democráticos, entre estas están firmar peticiones escritas con el objetivo de influir en la política; asistir a marchas, manifestaciones o movilizaciones colectivas legales y pacíficas; asistir a una reunión o debate político; colaborar con otras personas del barrio o sector para resolver problemas locales; donar dinero o recolectar fondos con fines políticos, contactar o aparecer en los medios de comunicación para expresar puntos de vista sobre asuntos políticos o de interés público; contactar o intentar ponerse en contacto con un político o funcionario público para expresar puntos de vista (sea personalmente o vía carta escrita). El comportamiento de protesta política se midió a través de 6 ítems que corresponden a las siguientes actividades políticas de carácter extrainstitucional: participar en huelgas o manifestaciones no autorizadas, rehusar pagar impuestos; bloquear el tráfico; participar en la ocupación de edificios públicos; boicotear o deliberadamente comprar ciertos productos por razones éticas, ambientales o económicas; tomar parte en protestas colectivas.

En el caso de la participación política online se incluyeron 14 ítems sobre conductas políticas individuales que pueden tener lugar en internet, entre estas: leer o publicar

2 Según estimaciones del año 2018, tomando en cuenta la población quiteña en edades de 20 años o más, una proporción aproximada del $26 \%$ correspondía al grupo de edad de 20 a 29 años. Mientras que, para la muestra, dicho grupo de edad representa alrededor del $49 \%$ del total de participantes con 20 años o más (Instituto Nacional de Estadísticas y Censos, 2018). 
comentarios en blogs, redes sociales o páginas web relacionadas con la política; contactar con algún político o funcionario público a través de internet; leer noticias de carácter político en los sitios web de medios tradicionales; ver videos de YouTube relacionados con temas u organizaciones políticas; escribir un blog o tomar parte en foros digitales sobre temas políticos; visitar páginas web relacionadas con partidos políticos, campañas electorales o temáticas políticas; enviar o reenviar correos electrónicos, audios o videos sobre cuestiones políticas; discutir con otros sobre política en internet; intentar persuadir a alguien para que apoye o se oponga a un proyecto político o candidato a través de internet; hacer trabajos, utilizando herramientas y aplicaciones de internet para promover un partido político, figura o iniciativa política; recolectar o contribuir con dinero a organizaciones o iniciativas políticas mediante internet; firmar o recolectar firmas para una petición o propuesta a través de internet; suscribirse a sitios web para recibir información de carácter político o de interés público.

Los ítems midieron la frecuencia de realización de cada actividad a partir de una escala de valoración de 5 puntos con las siguientes opciones de respuesta: 1 ("Nunca lo he hecho y nunca lo haría bajo ninguna circunstancia"), 2 ("No lo he hecho, pero podría hacerlo"), 3 ("Lo he hecho una o muy pocas veces"), 4 ("Lo he hecho algunas veces"), 5 ("Lo he hecho muchas veces"). Los puntajes de cada conjunto de ítems se sumaron para la construcción de índices aditivos de participación política convencional $[M=14,06$; DT $=5,48 ; \alpha=0,87]$ y protesta $[M=8,77 ; D T=4,12 ; \alpha=0,89]$ en el plano offline, así como de participación online $[M=26,06 ; \mathrm{DT}=10,12 ; \alpha=0,92]$.

\section{Variable independiente}

Para la medición del uso político de WhatsApp se contemplaron 8 ítems asociados con distintas actividades políticas que los usuarios pueden efectuar a través de esta aplicación móvil. Estas conductas se clasificaron en tres tipos: (a) consumo de información política mediante la recepción de imágenes, videos, enlaces, noticias u otros tipos de contenidos (1 ítem); (b) expresión de opiniones y discusión de asuntos políticos con otros usuarios, ya sea vía comentarios o respuestas a estados y publicaciones, compartiendo contenidos o manteniendo conversaciones por chat ${ }^{3}$ ( 4 ítems); y (c) creación, adhesión

3 Aunque la categoría de expresión política online suele abarcar tanto la publicación de posts como la conversación con otros usuarios (Rojas y Puig-i-Abril, 2009; Yamamoto et al., 2015), cabe resaltar la existencia de ciertas diferencias entre estos dos tipos de comportamientos. Por un lado, la publicación de puntos de vista a través de plataformas está orientada hacia la transmisión de mensajes a audiencias específicas, dependiendo de las listas de contactos del usuario. En redes sociales, estos posts pueden derivar o no en discusiones visibles públicamente como producto de comentarios posteados por otros usuarios. Sin embargo, AMI como WhatsApp permiten un mayor control de estas situaciones, impidiendo que los estados publicados puedan comentarse de manera pública. Por otra parte, la discusión interpersonal está centrada en el intercambio de ideas con otros usuarios, sea de manera individual o al interior de grupos online, teniendo desde un inicio una naturaleza 
o contacto con grupos formados para el tratamiento de cuestiones de interés público, asuntos electorales o la promoción de ideas y proyectos políticos (3 ítems). Cada ítem contó con una escala de valoración de 5 puntos con las siguientes opciones de respuesta: 1 ("Nunca"), 2 ("A veces"), 3 ("Normalmente"), 4 ("Casi siempre") y 5 ("Siempre"). Como resultado de la sumatoria de los ítems respectivos se obtuvieron índices aditivos para cada uso político de WhatsApp: consumo de información $[M=1,69 ; \mathrm{DT}=0,99]$, expresión y discusión política $[M=6,19 ; \mathrm{DT}=3,06 ; \alpha=0,90]$, y contacto con grupos políticos $[M=4,15$; $\mathrm{DT}=2,11 ; \alpha=0,88]$.

\section{Variables de control}

La selección de las variables de control se fundamentó en el modelo del voluntarismo cívico desarrollado por Verba et al. (1995), que es un aporte teórico utilizado frecuentemente por la literatura para explicar tanto la participación política offline como online. Según este modelo, la participación política se encuentra determinada por tres factores fundamentales: recursos, actitudes y reclutamiento. Los ingresos familiares y el nivel de educación actúan como indicadores de disponibilidad de recursos. Se contemplaron tres actitudes políticas: (a) la eficacia política interna se midió a partir de la escala de 4 ítems formulada por Niemi et al. (1991), considerando la adaptación efectuada por Zumárraga-Espinosa (2020) [ $M=10,30 ; D T=3,42 ; \alpha=0,81]$; (b) el interés en la política se cuantificó a través de un ítem con opciones de respuesta que varían desde 1 ("Nada interesado") hasta 4 ("Muy interesado") $[M=1.34$; DT $=0,91]$; (c) la ideología política se evaluó mediante una escala de autoposicionamiento que va desde 1 ("Izquierda") hasta 10 ("Derecha") [M = 5,28; DT = 2,09]. Por último, el contacto con los estímulos movilizadores producidos por organizaciones políticas y movimientos sociales se midió a través de la pertenencia a este tipo de agrupaciones. Al respecto se consultó la relación de los participantes con nueve agrupaciones de naturaleza política, como partidos políticos, sindicatos, organizaciones ambientalistas, entre otras. Para las respuestas se empleó una escala de valoración con las siguientes opciones de respuesta: 1 ("Nunca he sido miembro"), 2 ("Fui miembro en el pasado"), 3 ("Soy miembro, pero no participo"); 4 ("Soy miembro y participo activamente"). El índice de pertenencia a grupos políticos se calculó mediante la sumatoria de los puntajes de cada respuesta $[M=10,80 ; \mathrm{DT}=3,82 ; \alpha=0,89]$. El sexo y la edad también se incluyen como controles.

bidireccional. Sin embargo, para los fines de esta investigación, tanto la publicación de opiniones como la conversación política se conciben como parte de la misma variable "expresión/discusión", dado que implican la adopción de un rol activo en la comunicación política mediada por WhatsApp. 


\section{RESULTADOS}

Las preguntas de investigación se abordaron empíricamente a partir de regresiones jerárquicas por bloques. De esta manera se evalúa la relación entre los usos políticos de WhatsApp y cada modo de participación política incorporando el efecto producido por las variables de control. Asimismo, la construcción de modelos de regresión sucesivos permite desagregar la contribución explicativa realizada por cada componente del modelo del voluntarismo cívico y el bloque de conductas políticas correspondientes a esta AMI. La estimación de parámetros se efectúo a través del método de mínimos cuadrados ordinarios. La tabla 1 presenta las regresiones relativas a la participación política offline de tipo convencional. Se observa que el uso de WhatsApp para expresar y discutir cuestiones políticas se relaciona positivamente con la participación convencional $(\beta=0,263 ; p<0,001$ ), efecto movilizador que también se produce cuando esta plataforma se emplea para crear, seguir o interactuar con grupos de naturaleza política $(\beta=0,222 ; p<0,001)$. Contrariamente, el consumo de información política a través de WhatsApp no constituye un predictor significativo. Las variables de control explican el $27,6 \%$ de la variabilidad de la participación convencional, mientras que el peso explicativo de los usos políticos de WhatsApp se sitúa en $16,6 \%$.

Tabla 1

Análisis de regresión de la participación política offline, modalidad convencional

\begin{tabular}{|c|c|c|c|c|}
\hline & Modelo 1 & Modelo 2 & Modelo 3 & Modelo 4 \\
\hline \multicolumn{5}{|l|}{ Recursos/características demográficas } \\
\hline Sexo (masculino) & $0,063^{*}$ & 0,041 & 0,039 & 0,021 \\
\hline Edad & $-0,025$ & $-0,055^{*}$ & $-0,069^{* *}$ & $-0,009$ \\
\hline Ingresos familiares & $0,054^{*}$ & 0,015 & 0,020 & $-0,001$ \\
\hline Educación & 0,041 & $-0,010$ & $-0,003$ & 0,014 \\
\hline$R^{2}$ & $1,2 \%$ & & & \\
\hline \multicolumn{5}{|l|}{ Actitudes políticas } \\
\hline Eficacia política interna & & $0,234^{* * *}$ & $0,191^{* * *}$ & $0,139^{* * *}$ \\
\hline Interés en la política & & $0,215^{* * *}$ & $0,181^{* * *}$ & $0,119^{* * *}$ \\
\hline Ideología política & & $-0,026$ & $-0,039$ & $-0,039^{*}$ \\
\hline$R^{2}$ & & $13,5 \%$ & & \\
\hline \multicolumn{5}{|l|}{ Reclutamiento } \\
\hline Pertenencia a grupos políticos offline & & & $0,386^{* * *}$ & $0,181^{* * *}$ \\
\hline$R^{2}$ & & & $27,6 \%$ & \\
\hline \multicolumn{5}{|l|}{ Uso político de WhatsApp } \\
\hline Consumo de información & & & & 0,019 \\
\hline
\end{tabular}


(continuación)

\begin{tabular}{lc}
\hline Expresión/discusión & $0,263^{* * *}$ \\
Contacto con grupos políticos online & $0,222^{* * *}$ \\
$R^{2}$ & $44,2 \%$ \\
\hline
\end{tabular}

Nota. Se presentan los coeficientes de regresión estandarizados $(\beta) .{ }^{*} p<0,05 ;{ }^{* *} p<0,01$; ${ }^{* * *}$ $p<0,001$.

Elaboración propia, basada en los datos recolectados por el GIPS-UPS en Quito, Ecuador, entre junio y julio del 2018.

El análisis de regresión jerárquica correspondiente a la participación política offline de tipo protesta se muestra en la tabla 2. Los resultados sugieren que la protesta política se conecta significativamente con todos los usos políticos de WhatsApp contemplados en el estudio. Así, quienes emplean con mayor frecuencia esta plataforma para la revisión de contenidos políticos y noticias de interés público se encuentran más predispuestos a involucrarse en actividades de protesta política $(\beta=0,063 ; p<0,05)$. Situación similar ocurre con el uso de WhatsApp para la expresión de opiniones o la discusión política interpersonal $(\beta=0,100 ; p<0,05)$. Cabe resaltar que las conductas de WhatsApp asociadas con la creación, seguimiento y contacto con grupos políticos online son las que impactan con mayor fuerza sobre los comportamientos individuales de protesta $(\beta=0,285 ; p<0,001)$. La varianza explicada por el bloque de usos políticos de WhatsApp es del 12,7\%.

Tabla 2

Análisis de regresión de la participación política offline, modalidad protesta

\begin{tabular}{|c|c|c|c|c|}
\hline & Modelo 1 & Modelo 2 & Modelo 3 & Modelo 4 \\
\hline \multicolumn{5}{|l|}{ Recursos/características demográficas } \\
\hline Sexo (masculino) & $0,068^{* *}$ & $0,058^{*}$ & $0,055^{*}$ & $0,041^{*}$ \\
\hline Edad & $-0,109^{* * *}$ & $-0,121^{* * *}$ & $-0,137^{* * *}$ & $-0,086^{* * *}$ \\
\hline Ingresos familiares & $-0,016$ & $-0,040$ & $-0,034$ & $-0,055^{*}$ \\
\hline Educación & 0,026 & 0,005 & 0,014 & 0,029 \\
\hline$R^{2}$ & $1,8 \%$ & & & \\
\hline \multicolumn{5}{|l|}{ Actitudes políticas } \\
\hline Eficacia política interna & & $0,110^{* * *}$ & $0,057^{*}$ & 0,016 \\
\hline Interés en la política & & $0,119^{* * *}$ & $0,077^{* *}$ & 0,030 \\
\hline Ideología política & & 0,010 & $-0,007$ & $-0,009$ \\
\hline$R^{2}$ & & $5,1 \%$ & & \\
\hline \multicolumn{5}{|l|}{ Reclutamiento } \\
\hline Pertenencia a grupos políticos offline & & & $0,473^{* * *}$ & $0,291^{* * *}$ \\
\hline$R^{2}$ & & & $26,8 \%$ & \\
\hline
\end{tabular}


(continuación)

\begin{tabular}{lc} 
Consumo de información & $0,063^{*}$ \\
Expresión/discusión & $0,100^{*}$ \\
Contacto con grupos políticos online & $0,285^{* * *}$ \\
$R^{2}$ & $39,5 \%$ \\
\hline
\end{tabular}

Nota. Se presentan los coeficientes de regresión estandarizados $(\beta) .{ }^{*} p<0,05 ;{ }^{* *} p<0,01 ;{ }^{* * *}$ $p<0,001$.

Elaboración propia, basada en datos recolectados por el GIPS-UPS en Quito, Ecuador, entre junio y julio del 2018.

Los resultados con respecto a la participación política online son similares a aquellos obtenidos en el caso de la participación offline de tipo convencional (véase la tabla 3). El uso informacional de WhatsApp no se asocia de modo significativo con la participación online. Por su parte, los usos expresivos $(\beta=0,288 ; p<0,001)$ y de contacto con grupos políticos online $(\beta=0,219 ; p<0,001)$ efectivamente influyen sobre las conductas políticas adoptadas de forma general a través de internet. Sin embargo, las acciones centradas en la expresión y la discusión sobre tópicos políticos o de interés público muestran un efecto movilizador ligeramente superior. En su conjunto, los predictores empleados tienen mayor capacidad para explicar la participación online $\left(R^{2}=48,0 \%\right)$ que la participación offline en su modalidad convencional $\left(R^{2}=44,2 \%\right)$ o protesta $\left(R^{2}=39,5 \%\right)$. Esto también aplica para los usos políticos de WhatsApp, contribuyendo a explicar el 18,9\% de las variaciones de la participación política online.

Tabla 3

Análisis de regresión de la participación política online

\begin{tabular}{|c|c|c|c|c|}
\hline & Modelo 1 & Modelo 2 & Modelo 3 & Modelo 4 \\
\hline \multicolumn{5}{|c|}{ Recursos/características demográficas } \\
\hline Sexo (masculino) & $0,052^{*}$ & 0,035 & 0,033 & 0,014 \\
\hline Edad & $-0,148^{* * *}$ & $-0,164^{* * *}$ & $-0,175^{* * *}$ & $-0,110^{* * *}$ \\
\hline Ingresos familiares & 0,043 & $-0,003$ & 0,000 & $-0,022$ \\
\hline Educación & $0,094^{* *}$ & 0,036 & 0,041 & $0,059^{* *}$ \\
\hline$R^{2}$ & $4,2 \%$ & & & \\
\hline \multicolumn{5}{|l|}{ Actitudes políticas } \\
\hline Eficacia política interna & & $0,184^{* * *}$ & $0,148^{* * *}$ & $0,094^{* * *}$ \\
\hline Interés en la política & & $0,306^{* * *}$ & $0,278^{* * *}$ & $0,211^{* * *}$ \\
\hline Ideología política & & 0,019 & 0,008 & 0,009 \\
\hline$R^{2}$ & & $19,4 \%$ & & \\
\hline
\end{tabular}


(continuación)

\begin{tabular}{|c|c|c|}
\hline \multicolumn{3}{|l|}{ Reclutamiento } \\
\hline Pertenencia a grupos políticos offline & $0,317^{* * *}$ & $0,100^{* * *}$ \\
\hline$R^{2}$ & $29,1 \%$ & \\
\hline \multicolumn{3}{|l|}{ Uso político de WhatsApp } \\
\hline Consumo de información & & 0,030 \\
\hline Expresión/discusión & & $0,288^{* * *}$ \\
\hline Contacto con grupos políticos online & & $0,219^{* * *}$ \\
\hline$R^{2}$ & & $48,0 \%$ \\
\hline
\end{tabular}

Nota. Se presentan los coeficientes de regresión estandarizados $(\beta)^{*} p<0.05 ;{ }^{* *} p<0,01$; ${ }^{* *}$ $p<0,001$.

Elaboración propia, basada en los datos recolectados por el GIPS-UPS en Quito, Ecuador, entre junio y julio del 2018.

\section{DISCUSIÓN}

La investigación efectuada tiene el propósito de aportar conocimiento con respecto a los efectos democráticos de la comunicación política móvil vía AMI, abordando específicamente el caso de WhatsApp en el contexto ecuatoriano. Los hallazgos empíricos muestran que el uso político de WhatsApp ejerce efectos movilizadores sobre la participación política en todas las dimensiones analizadas: offline convencional, offline protesta y online. No obstante, existen particularidades al desagregar dicha influencia según el tipo de conductas políticas desarrolladas a través de esta aplicación. A diferencia de su uso para el consumo de información política (que solo conecta con la participación offline protesta), los usos centrados en la expresión de opiniones y la discusión política interpersonal impactan de manera consistente sobre todas las dimensiones de la participación política. Esto, además de coincidir con hallazgos reportados previamente en la limitada literatura sobre el uso político de WhatsApp (Gil de Zúñiga et al., 2019; Valenzuela et al., 2019), respaldaría los planteamientos teóricos del modelo de mediación cognitiva propuesto por Eveland (2001). Según este modelo, aspectos como la atención y el procesamiento de información son condicionantes clave para que los actos individuales de comunicación política fomenten efectivamente el aprendizaje político, lo cual a su vez promueve la participación política. En cuanto a efectos de la discusión política, Eveland (2004) concluye que tanto las elaboraciones mentales previas como aquellas que emergen al momento de discutir con otros influyen positivamente sobre el conocimiento político, al obtenerse menor respaldo empírico al evaluar únicamente la exposición a información política producto del intercambio de argumentos y puntos de vista. 
Conjuntamente, el efecto movilizador aportado por la expresión de puntos de vista y opiniones políticas puede interpretarse a la luz del modelo de "efectos bidireccionales del mensaje" de Pingree (2007), el cual sostiene que cuando el individuo pasa de receptor a emisor de mensajes políticos se desencadena una serie de mecanismos que puede estimular no solo el aprendizaje político, sino también actitudes y estados emocionales más favorables para la participación política. Tales mecanismos van desde la expectativa previa a la expresión, que refuerza la atención hacia la información recibida y su retención en la memoria para ser procesada al momento de formular opiniones, hasta los esfuerzos de composición que demandan replantear ideas y creencias, así como explorar nueva información, a fin de construir argumentos más robustos. El mensaje una vez emitido puede alterar la autopercepción política del individuo, pues los puntos de vista expresados pasan a integrar su autoconcepto, además de crear compromiso en torno a lo expresado por razones de coherencia. Al respecto, los hallazgos de Lee y Hsieh (2013) proponen la existencia de un efecto de consistencia entre las conductas políticas online y la posterior implicación en acciones participativas del mundo real. En otras palabras, cuando un usuario realiza actos políticos online asociados con algún tema o problemática específica, la necesidad de coherencia aumenta su predisposición a participar en actividades políticas offline sobre tópicos similares. Los argumentos expuestos sugieren que los procesos mentales conducentes hacia los comportamientos participativos se desarrollan en mayor medida cuando los individuos pasan de ser receptores pasivos de información a desempeñar un rol más activo en el proceso de comunicación política. Esto explicaría la estabilidad de los efectos movilizadores producidos por el uso de WhatsApp para la expresión y la discusión política.

Los resultados expuestos amplían la literatura sobre comunicación móvil, AMI y participación política al explorar los efectos democráticos de una plataforma relativamente poco estudiada hasta el momento como WhatsApp. Así, también se ha buscado profundizar en la relación entre las formas de participación política y las funciones de creación, seguimiento o contacto con grupos políticos ofrecidas por WhatsApp. Tópico que previamente solo había sido analizado en el caso de Facebook (Conroy et al., 2012; Valenzuela et al., 2009). Como pudo observarse, si bien el uso de WhatsApp para la interacción con grupos formados para el tratamiento de cuestiones políticas favorece todos los modos de participación contemplados, este efecto se intensifica cuando se trata de las actividades de protesta política. Cambio de patrón que adquiere sentido, puesto que factores como la construcción de experiencias colectivas y el acceso a información movilizadora poseen especial relevancia para la protesta política (Valenzuela et al., 2012; Valenzuela, 2013). En este sentido, la capacidad de WhatsApp para generar sentido de comunidad (Church y De Oliveira, 2013) facilita la construcción de identidades colectivas en torno a cuestiones políticas concretas. Asimismo, la interconexión entre grupos políticos de WhatsApp y otros grupos online u offline que comparten intereses comunes eleva la probabilidad de entrar en contacto con información movilizadora, por 
ejemplo, agendas de movilización, cronogramas, lugares de encuentro, convocatorias o peticiones para participar en acciones colectivas de protesta. De manera general los grupos políticos online también operan como espacios estimulantes para la expresión y la discusión política interpersonal entre sus miembros (Conroy et al., 2012), lo cual puede potenciar la relación entre este tipo de conductas y la participación política.

Por último, los grupos online, como herramientas de los medios sociales, posibilitan que los usuarios se mantengan al tanto sobre lo que ocurre con el resto de miembros; además, el intercambio de información permanente promueve la confianza interpersonal, cuyo resultado es un incremento del capital social (Gil de Zúñiga et al., 2012). Así, los hallazgos de Valenzuela et al. (2009) evidencian que la frecuencia con la que los usuarios interactúan con grupos de Facebook se asocia positivamente con sus niveles de capital social. A su vez, el capital social es un predictor importante de la participación política (Gil de Zúñiga et al., 2012; Klesner, 2007). Dada la preferencia que las personas suelen asignar a WhatsApp como medio para interactuar con otros de manera directa, así como mantener un contacto más continuo y cercano con su círculo social (O'Hara et al., 2014), podría esperarse que los grupos políticos creados a través de esta aplicación produzcan beneficios aún mayores en términos de capital social. Adicionalmente, resulta interesante señalar que la interacción con grupos políticos de WhatsApp y la pertenencia a grupos políticos offline ejercen efectos diferenciados sobre las formas de participación política, lo cual sugiere que la actividad al interior de agrupaciones políticas digitales cuenta con capacidad propia para favorecer la movilización política de sus miembros.

Resta decir que la intermitencia observada en los efectos movilizadores del consumo de información puede deberse a las características propias de WhatsApp, que es un espacio un tanto más limitado para compartir de forma detallada contenidos y noticias políticas, en comparación con Facebook o Twitter. No obstante, el hecho de que el único efecto significativo reportado corresponda a la modalidad de protesta apoya la idea de que WhatsApp, al configurar un espacio de comunicación más controlado, funciona como un medio más atractivo para la circulación de información movilizadora asociada con actividades políticas contenciosas (Gil de Zúñiga et al., 2019).

Una limitación del estudio corresponde al uso de datos de corte transversal, por lo que se requieren posteriores investigaciones basadas en información longitudinal o diseños experimentales para confirmar de manera rigurosa la orientación causal de los resultados aquí expuestos, especialmente en cuanto a los hallazgos que no han sido previamente contemplados por la literatura sobre WhatsApp y sus implicaciones políticas. Además, considerando el perfil de la muestra utilizada, es probable que los resultados tengan mayor aplicabilidad para la población ecuatoriana más joven, y sea necesario realizar nuevos estudios con muestras de representatividad poblacional. Complementariamente, quedan por evaluar las condiciones y mecanismos que hacen posible que cada uso político de WhatsApp influya sobre los comportamientos de 
participación política en sus respectivas modalidades; en tal sentido, se sugiere la exploración de variables moderadoras y mediadoras.

\section{REFERENCIAS}

Baek, Y. M. (2015). Political mobilization through social network sites: the mobilizing power of political messages received from SNS friends. Computers in Human Behavior, 44, 12-19. https://doi.org/10.1016/j.chb.2014.11.021

Bakshy, E., Messing, S., y Adamic, L. A. (2015). Exposure to ideologically diverse news and opinion on Facebook. Science, 348(6239), 1130-1132. https://doi.org/10.1126/ science.aaa1160

Boulianne, S. (2015). Social media use and participation: a meta-analysis of current research. Information, Communication \& Society, 18(5), 524-538. https://doi.org/ 10.1080/1369118X.2015.1008542

Boulianne, S. (2020). Twenty years of digital media effects on civic and political participation. Communication Research, 47(7), 947-966. https://doi. org/10.1177/0093650218808186

Boulianne, S., y Theocharis, Y. (2020). Young people, digital media, and engagement: a meta-analysis of research. Social Science Computer Review, 38(2), 111-127. https://doi.org/10.1177/0894439318814190

Campbell, S. W., y Kwak, N. (2010). Mobile communication and civic life: linking patterns of use to civic and political engagement. Journal of Communication, 60(3), 536-555. https://doi.org/10.1111/j.1460-2466.2010.01496.x

Campbell, S. W., y Kwak, N. (2011). Political involvement in "mobilized" society: the interactive relationships among mobile communication, network characteristics, and political participation. Journal of Communication, 61(6), 1005-1024. https:// doi.org/10.1111/j.1460-2466.2011.01601.x

Chan, M., Chen, H. T., y Lee, F. L. (2017). Examining the roles of mobile and social media in political participation: a cross-national analysis of three Asian societies using a communication mediation approach. New Media \& Society, 19(12), 2003-2021. https://doi.org/10.1177/1461444816653190

Cho, J., Shah, D. V., McLeod, J. M., McLeod, D. M., Scholl, R. M., y Gotlieb, M. R. (2009). Campaigns, reflection, and deliberation: advancing an O-S-R-O-R model of communication effects. Communication Theory, 19(1), 66-88. https://doi. org/10.1111/j.1468-2885.2008.01333.x

Church, K., y De Oliveira, R. (2013). What's up with WhatsApp?: comparing mobile instant messaging behaviors with traditional SMS. En Proceedings of the 15th 
International Conference on Human-Computer Interaction with Mobile Devices and Services (pp. 352-361). https://dl.acm.org/citation.cfm?id=2493225

Conroy, M., Feezell, J. T., y Guerrero, M. (2012). Facebook and political engagement: a study of online political group membership and offline political engagement. Computers in Human Behavior, 28(5), 1535-1546. https://doi.org/10.1016/j. chb.2012.03.012

DiGrazia, J. (2014). Individual protest participation in the United States: conventional and unconventional activism. Social Science Quarterly, 95(1), 111-131. https://doi. org/10.1111/ssqu. 12048

Druckman, J. N., y Nelson, K. R. (2003). Framing and deliberation: how citizens' conversations limit elite influence. American Journal of Political Science, 47(4), 729-745. https://doi.org/10.1111/1540-5907.00051

Effing, R., Van Hillegersberg, J., y Huibers, T. (2011). Social media and political participation: are Facebook, Twitter and YouTube democratizing our political systems? En International Conference on Electronic Participation (pp. 25-35). Springer. https:// doi.org/10.1007/978-3-642-23333-3_3

Ekman, J, y Amnå, E. (2012). Political participation and civic engagement: towards a new typology. Human Affairs, 22(3), 283-300. https://doi.org/10.2478/s13374-0120024-1

Eveland, W. P. (2001). The cognitive mediation model of learning from the news: evidence from nonelection, off-year election, and presidential election contexts. Communication Research, 28(5), 571-601. https://doi.org/10.1177/009365001028 005001

Eveland, W. P. (2004). The effect of political discussion in producing informed citizens: the roles of information, motivation, and elaboration. Political Communication, 21(2), 177-193. https://doi.org/10.1080/10584600490443877

Fenton, N., y Barassi, V. (2011). Alternative media and social networking sites: the politics of individuation and political participation. The Communication Review, 14(3), 179-196. https://doi.org/10.1080/10714421.2011.597245

Gazit, T., y Aharony, N. (2018). Factors explaining participation in WhatsApp groups: an exploratory study. Aslib Journal of Information Management, 70(4), 390-413. https://doi.org/10.1108/AJIM-03-2018-0053

Gibson, R., y Cantijoch, M. (2013). Conceptualizing and measuring participation in the age of the internet: is online political engagement really different to offline? The Journal of Politics, 75(3), 701-716. https://doi.org/10.1017/S0022381613000431 
Gil de Zúñiga, H., Ardèvol-Abreu, A., y Casero-Ripollés, A. (2019). WhatsApp political discussion, conventional participation and activism: exploring direct, indirect and generational effects. Information, Communication \& Society, 24(2), 201-218. https://doi.org/10.1080/1369118X.2019.1642933

Gil de Zúñiga, H., Jung, N., y Valenzuela, S. (2012). Social media use for news and individuals' social capital, civic engagement and political participation. Journal of Computer-Mediated Communication, 17(3), 319-336. https://doi.org/10.1111/ j.1083-6101.2012.01574.x

Gil de Zúñiga, H., Molyneux, L., y Zheng, P. (2014). Social media, political expression, and political participation: panel analysis of lagged and concurrent relationships. Journal of Communication, 64(4), 612-634. https://doi.org/10.1111/jcom.12103

Gil de Zúñiga, H., y Valenzuela, S. (2011). The mediating path to a stronger citizenship: online and offline networks, weak ties, and civic engagement. Communication Research, 38(3), 397-421. https://doi.org/10.1177/0093650210384984

Hardy, B. W., y Scheufele, D. A. (2005). Examining differential gains from Internet use: comparing the moderating role of talk and online interactions. Journal of Communication, 55(1), 71-84. https://doi.org/10.1111/j.1460-2466.2005.tb02659.x

Hollander, B. A. (1995). The influence of talk radio on political efficacy and participation. Journal of Radio Studies, 3(1), 23-31. https://doi.org/10.1080/19376529509361971

Hopke, J. E., Gabay, I., Kim, S. C., y Rojas, H. (2016). Mobile phones and political participation in Colombia: mobile Twitter versus mobile Facebook. Communication and the Public, 1(2), 159-173. https://doi.org/10.1177/2057047316642607

Instituto Nacional de Estadísticas y Censos (2018). Proyecciones poblacionales [fichero de datos]. https://bit.ly/2QEfkDm

Jung, N., Kim, Y., y Gil de Zúñiga, H. (2011). The mediating role of knowledge and efficacy in the effects of communication on political participation. Mass Communication and Society, 14(4), 407-430. https://doi.org/10.1080/15205436.2010.496135

Karapanos, E., Teixeira, P., y Gouveia, R. (2016). Need fulfillment and experiences on social media: a case on Facebook and WhatsApp. Computers in Human Behavior, 55(Part B), 888-897. https://doi.org/10.1016/j.chb.2015.10.015

Katz, E., y Gurevitch, M. (1974). Utilization of mass communication by the individual. En J. Blumler y E. Katz (Eds.), The uses of mass communication: current perspectives on gratifications research (pp. 19-32). Sage Publications.

Kim, Y., Russo, S., y Amnå, E. (2017). The longitudinal relation between online and offline political participation among youth at two different developmental stages. New Media \& Society, 19(6), 899-917. https://doi.org/10.1177/1461444815624181 
Klesner, J. L. (2007). Social capital and political participation in Latin America: evidence from Argentina, Chile, Mexico, and Peru. Latin American Research Review, 42(2), 1-32. https://muse.jhu.edu/article/218046/summary

Krueger, B. S. (2002). Assessing the potential of Internet political participation in the United States: a resource approach. American Politics Research, 30(5), 476-498. https://doi.org/10.1177/1532673X02030005002

Lee, Y. H., y Hsieh, G. (2013). Does slacktivism hurt activism?: the effects of moral balancing and consistency in online activism. En Proceedings of the SIGCHI Conference on Human Factors in Computing Systems (pp. 811-820). https://dl.acm.org/citation.cfm?id=2470770

Lupia, A., y Sin, G. (2003). Which public goods are endangered?: how evolving communication technologies affect the logic of collective action. Public Choice, 117(3-4), 315-331. https://doi.org/10.1023/B:PUCH.0000003735.07840.c7

Moncagatta, P., Moreno, A. M., Pachano, S., Montalvo, J. D., y Zechmeister, E. J. (2020). Cultura política de la democracia en Ecuador y en las Américas, 2018/19: tomándole el pulso a la democracia. Agencia de los Estados Unidos para el Desarrollo Internacional (USAID).

Montag, C., Błaszkiewicz, K., Sariyska, R., Lachmann, B., Andone, I., Trendafilov, B., ... Markowetz, A. (2015). Smartphone usage in the 21st century: who is active on WhatsApp? BMC Research Notes, 8(1), 331. https://doi.org/10.1186/s13104-015$1280-z$

Mutz, D. C. (2002). The consequences of cross-cutting networks for political participation. American Journal of Political Science, 46(4), 838-855. https://www.jstor.org/ stable/3088437

Nam, T. (2012). Dual effects of the internet on political activism: reinforcing and mobilizing. Government Information Quarterly, 29, S90-S97. https://doi.org/10.1016/j. giq.2011.08.010

Newman, N., Fletcher, R., Kalogeropoulos, A., Levy D., y Nielsen, R. K. (2018). Reuters Institute Digital News Report 2018. The Reuters Institute for the Study of Journalism.

Niemi, R. G., Craig, S. C., y Mattei, F. (1991). Measuring internal political efficacy in the 1988 National Election Study. The American Political Science Review, 85(4), 1407-1413. https://doi.org/10.2307/1963953

O'Hara, K. P., Massimi, M., Harper, R., Rubens, S., y Morris, J. (2014). Everyday dwelling with WhatsApp. En Proceedings of the 17th ACM Conference on Computer Supported Cooperative Work \& Social Computing (pp. 1131-1143). https://dl.acm.org/citation. cfm?id=2531679 
Oñate, P. (2005). Participación política, partidos y nuevos movimientos sociales. Revista Mexicana de Ciencias Políticas y Sociales, 47(194), 103-135. http://dx.doi. org/10.22201/fcpys.2448492xe.2005.194.42488

Oser, J., Hooghe, M., y Marien, S. (2013). Is online participation distinct from offline participation? A latent class analysis of participation types and their stratification. Political Research Quarterly, 66(1), 91-101. https://doi.org/10.1177/1065912912436695

Pang, H. (2018). Mobile communication and political participation: unravelling the effects of mobile phones on political expression and offline participation among young people. International Journal of Electronic Governance, 10(1), 3-23. https://doi. org/10.1504/IJEG.2018.091262

Park, C. S., y Karan, K. (2014). Unraveling the relationships between smartphone use, exposure to heterogeneity, political efficacy, and political participation: a mediation model approach. Asian Journal of Communication, 24(4), 370-389. https://doi. org/10.1080/01292986.2014.892146

Pingree, R. J. (2007). How messages affect their senders: a more general model of message effects and implications for deliberation. Communication Theory, 17(4), 439-461. https://doi.org/10.1111/j.1468-2885.2007.00306.x

Prendes-Espinosa, M., Gutiérrez-Porlán, I., y Castañeda-Quintero, L. (2015). Perfiles de uso de redes sociales: estudio descriptivo con alumnado de la Universidad de Murcia. Revista Complutense de Educación, 26, 75-195. http://dx.doi.org/10.5209/ RCED

Quan-Haase, A., y Young, A. L. (2010). Uses and gratifications of social media: a comparison of Facebook and instant messaging. Bulletin of Science, Technology \& Society, 30(5), 350-361. https://doi.org/10.1177/0270467610380009

Rojas, H., y Puig-i-Abril, E. (2009). Mobilizers mobilized: information, expression, mobilization and participation in the digital age. Journal of Computer-Mediated Communication, 14(4), 902-927. https://doi.org/10.1111/j.1083-6101.2009.01475.x

Rubio-Romero, J., y Perlado, M. (2015). El fenómeno WhatsApp en el contexto de la comunicación personal: una aproximación a través de los jóvenes universitarios. ICONO14. Revista científica de Comunicación y Tecnologías Emergentes, 13(2), 73-94. https://doi.org/10.7195/ri14.v13i2.818

Ruggiero, T.E.(2000). Uses and gratifications theory in the 21stcentury. Mass Communication \& Society, 3(1), 3-37. https://doi.org/10.1207/S15327825MCS0301_02

Ruiz-Corbella, M., y Juanas-Oliva, Á. (2013). Redes sociales, identidad y adolescencia: nuevos retos educativos para la familia. Estudios sobre Educación, 25, 95-113. http://hdl.handle.net/10171/34734 
Scheufele, D. A., y Nisbet, M. C. (2002). Being a citizen online: new opportunities and dead ends. Harvard International Journal of Press/Politics, 7(3), 55-75. https://doi. org/10.1177/1081180X0200700304

Shah, D. V., Cho, J., Eveland, W. P., y Kwak, N. (2005). Information and expression in a digital age: modeling Internet effects on civic participation. Communication Research, 32(5), 531-565. https://doi.org/10.1177/0093650205279209

Shah, D. V., Cho, J., Nah, S., Gotlieb, M. R., Hwang, H., Lee, N. J., Scholl, R., y McLeod, D. M. (2007). Campaign ads, online messaging, and participation: extending the communication mediation model. Journal of Communication, 57(4), 676-703. https://doi.org/10.1111/j.1460-2466.2007.00363.x

Shah, D. V., Kwak, N., y Holbert, L. R. (2001). "Connecting" and "disconnecting" with civic life: patterns of Internet use and the production of social capital. Political Communication, 18(2), 141-162. https://doi.org/10.1080/105846001750322952

Skoric, M. M., y Zhu, Q. (2016). Social media and offline political participation: uncovering the paths from digital to physical. International Journal of Public Opinion Research, 28(3), 415-427. https://doi.org/10.1093/ijpor/edv027

Skoric, M. M., Zhu, Q., Goh, D., y Pang, N. (2016). Social media and citizen engagement: a meta-analytic review. New Media \& Society, 18(9), 1817-1839. https://doi. org/10.1177/1461444815616221

Somuano, M. F. (2005). Más allá del voto: modos de participación política no electoral en México. Foro Internacional, 45(1), 65-88. https://www.jstor.org/stable/27738690

Theocharis, Y., Lowe, W., Van Deth, J. W., y García-Albacete, G. (2015). Using Twitter to mobilize protest action: online mobilization patterns and action repertoires in the Occupy Wall Street, Indignados, and Aganaktismenoi movements. Information, Communication \& Society, 18(2), 202-220. https://doi.org/10.1080/13 69118X.2014.948035

Valenzuela, S. (2013). Unpacking the use of social media for protest behavior: the roles of information, opinion expression, and activism. American Behavioral Scientist, 57(7), 920-942. https://doi.org/10.1177/0002764213479375

Valenzuela, S., Arriagada, A., y Scherman, A. (2012). The social media basis of youth protest behavior: the case of Chile. Journal of Communication, 62(2), 299-314. https://doi.org/10.1111/j.1460-2466.2012.01635.x

Valenzuela, S., Bachmann, I., y Bargsted, M. (2019). The personal is the political? What do WhatsApp users share and how it matters for news knowledge, polarization and participation in Chile. Digital Journalism, 9(2), 1-21. https://doi.org/10.1080/2167 0811.2019 .1693904 
Valenzuela, S., Park, N., y Kee, K. F. (2009). Is there social capital in a social network site?: Facebook use and college students' life satisfaction, trust, and participation. Journal of Computer-Mediated Communication, 14(4), 875-901. https://doi. org/10.1111/j.1083-6101.2009.01474.x

Valeriani, A., y Vaccari, C. (2018). Political talk on mobile instant messaging services: a comparative analysis of Germany, Italy, and the UK. Information, Communication \& Society, 21(11), 1715-1731. https://doi.org/10.1080/1369118X.2017.1350730

Verba, S., Schlozman, K. L., y Brady, H. E. (1995). Voice and equality. Civic voluntarism in American politics. Harvard University Press.

We Are Social y Hootsuite. (2020). Digital in 2020. Global Digital Overview. https://wearesocial.com/digital-2020

WhatsApp. (20 de agosto del 2020). Funciones. https://www.whatsapp.com/features/

Wojcieszak, M. (2009). "Carrying online participation offline" - Mobilization by radical online groups and politically dissimilar offline ties. Journal of Communication, 59(3), 564-586. https://doi.org/10.1111/j.1460-2466.2009.01436.x

Yamamoto, M., Kushin, M. J., y Dalisay, F. (2015). Social media and mobiles as political mobilization forces for young adults: examining the moderating role of online political expression in political participation. New Media \& Society, 17(6), 880-898. https://doi.org/10.1177/1461444813518390

Yang, H. C., y DeHart, J. L. (2016). Social media use and online political participation among college students during the US election 2012. Social Media + Society, 2(1), 1-18.

Zumárraga-Espinosa, M. (2020). Aportes metodológicos para la medición del sentido de eficacia política: evidencia empírica de Quito-Ecuador. Empiria: Revista de Metodología de Ciencias Sociales, (45), 113-142. https://doi.org/10.5944/ empiria.45.2020.26306

Zumárraga-Espinosa, M., Reyes, C., y Carofilis, C. (2017). ¿Verdad o ficción? El uso político de las redes sociales en la participación política offline en las elecciones presidenciales en Ecuador. Análisis Político, 30(91), 130-145. https://doi.org/10.15446/ anpol.v30n91.70268 